\title{
The inverses of tails of the Riemann zeta function
}

\section{Donggyun $\mathrm{Kim}^{1}$ (D) and Kyunghwan Song ${ }^{1 *}$ (D)}

"Correspondence:

heroesof@korea.ac.kr

${ }^{1}$ Department of Mathematics, Korea

University, Seoul, Republic of Korea

\begin{abstract}
We present some bounds of the inverses of tails of the Riemann zeta function on $0<s<1$ and compute the integer parts of the inverses of tails of the Riemann zeta function for $s=\frac{1}{2}, \frac{1}{3}$, and $\frac{1}{4}$.
\end{abstract}

MSC: Riemann zeta function; Tails of Riemann zeta function; Inverses of tails of the Riemann zeta function

Keywords: $11 \mathrm{M} 06 ; 11 \mathrm{~B} 83$

\section{Introduction}

The Riemann zeta function $\zeta(s)$ in the real variable $s$ was introduced by Euler [2] in connection with questions about the distribution of prime numbers. Later Riemann [6] derived deeper results about a dual correspondence between the distribution of prime numbers and the complex zeros of $\zeta(s)$ in the complex variable $s$. In these developments, he asserted that all the non-trivial zeros of $\zeta(s)$ are on the line $\operatorname{Re}(s)=\frac{1}{2}$, and this has been one of the most important unsolved problems in mathematics, called the Riemann hypothesis. A vast amount of research on calculation of $\zeta(s)$ on the line $\operatorname{Re}(s)=\frac{1}{2}$, which is called the critical line, and on the strip $0<\operatorname{Re}(s)<1$, which is called the critical strip, has been conducted using various methods [1].

The Riemann zeta function and a tail of the Riemann zeta function from $n$ for an integer $n \geq 1$ are defined, respectively, by: for $\operatorname{Re}(s)>1$,

$$
\zeta(s)=\sum_{k=1}^{\infty} \frac{1}{k^{s}} \quad \text { and } \quad \zeta_{n}(s)=\sum_{k=n}^{\infty} \frac{1}{k^{s}}
$$

and for $0<\operatorname{Re}(s)<1$,

$$
\zeta(s)=\frac{1}{1-2^{1-s}} \sum_{k=1}^{\infty} \frac{(-1)^{k+1}}{k^{s}} \quad \text { and } \quad \zeta_{n}(s)=\frac{1}{1-2^{1-s}} \sum_{k=n}^{\infty} \frac{(-1)^{k+1}}{k^{s}}
$$

To understand the values of $\zeta(s)$, it would be helpful to understand the values of tails of $\zeta(s)$, for example, the integer parts of their inverses $\left[\zeta_{n}(s)^{-1}\right]$, where $[x]$ denotes the greatest integer that is less than or equal to $x$.

(c) The Author(s) 2018. This article is distributed under the terms of the Creative Commons Attribution 4.0 International License (http://creativecommons.org/licenses/by/4.0/), which permits unrestricted use, distribution, and reproduction in any medium, provided you give appropriate credit to the original author(s) and the source, provide a link to the Creative Commons license, and indicate if changes were made. 
Some values of $\left[\zeta_{n}(s)^{-1}\right]$ for small positive integers $s$ have become known recently. Xin [7] showed that for $s=2$ and 3,

$$
\left[\zeta_{n}(2)^{-1}\right]=n-1 \quad \text { and } \quad\left[\zeta_{n}(3)^{-1}\right]=2 n(n-1)
$$

For $s=4$, Xin and Xiaoxue [8] showed that

$$
\left[\zeta_{n}(4)^{-1}\right]=3 n^{3}-5 n^{2}+4 n-1+\left[\frac{(2 n+1)(n-1)}{4}\right]
$$

for any integer $n \geq 2$, and $\mathrm{Xu}[9]$ showed that for $s=5$,

$$
\left[\zeta_{n}(5)^{-1}\right]=4 n^{4}-8 n^{3}+9 n^{2}-5 n+\left[\frac{(n+1)(n-2)}{3}\right]
$$

for any integer $n \geq 4$. Hwang and Song [3] provided an alternative proof of the case when $s=5$ and a formula when $s=6$ as follows. For an integer $n$, write $n_{48}$ for the remainder when $n$ is divided by 48 , then

$$
\begin{aligned}
& {\left[\zeta_{n}(6)^{-1}\right]} \\
& \qquad \begin{cases}5 n^{5}-\frac{25}{2} n^{4}+\frac{75}{4} n^{3}-\frac{125}{8} n^{2}+\frac{185}{48} n-\frac{5 n_{48}}{48}-\left[\frac{35-5 n_{48}}{48}\right], & \text { if } n \text { is even, } \\
5 n^{5}-\frac{25}{2} n^{4}+\frac{75}{4} n^{3}-\frac{125}{8} n^{2}+\frac{185}{48} n-\frac{5 n_{48}+18}{48}-\left[\frac{17-5 n_{48}}{48}\right], & \text { if } n \text { is odd }\end{cases}
\end{aligned}
$$

for any integer $n \geq 829$. For the integer $s$ greater than 6 , no such a formula is known.

There are other interesting results related to this theme such as bounds of $\zeta(3)$ in greater precision in [4] and [5].

We study the inverses of tails of the Riemann zeta function $\zeta_{n}(s)^{-1}$ for $s$ on the critical strip $0<s<1$. The following notation is needed to explain our results.

Definition 1 For any positive integer $n$ and real number $s$ with $0<s<1$, we define

$$
A_{n, s}=\left(\frac{1}{n^{s}}-\frac{1}{(n+1)^{s}}\right)+\left(\frac{1}{(n+2)^{s}}-\frac{1}{(n+3)^{s}}\right)+\cdots
$$

and

$$
B_{n, s}=\left(-\frac{1}{n^{s}}+\frac{1}{(n+1)^{s}}\right)+\left(-\frac{1}{(n+2)^{s}}+\frac{1}{(n+3)^{s}}\right)+\cdots .
$$

Now the tail of the Riemann zeta function for $0<s<1$ can be written as follows:

$$
\zeta_{n}(s)= \begin{cases}-\frac{1}{1-2^{1-s}} A_{n, s}, & \text { if } n \text { is even, } \\ -\frac{1}{1-2^{1-s}} B_{n, s}, & \text { if } n \text { is odd. }\end{cases}
$$

In this paper, we present the bounds of $A_{h, s}^{-1}$ and $B_{h, s}^{-1}$, hence the bounds of the inverses of tails of the Riemann zeta function $\zeta_{n}(s)^{-1}$ for $0<s<1$ in Sect. 2.1, and compute the values $\left[A_{n, s}^{-1}\right]$ and $\left[B_{n, s}^{-1}\right]$, hence the values of the inverses of tails of the Riemann zeta function $\left[\frac{1}{1-2^{1-s}} \zeta_{n}(s)^{-1}\right]$ for $s=\frac{1}{2}, \frac{1}{3}$, and $\frac{1}{4}$ in Sect. 2.2. 


\section{Main results}

\subsection{The bounds of the inverses of $\zeta_{n}(s)$ for $0<s<1$}

In this section, we present the bounds of $A_{n, s}^{-1}$ and $B_{n, s}^{-1}$ in Definition 1, hence the bounds of the inverses of tails of the Riemann zeta function $\zeta_{n}(s)^{-1}$ for $0<s<1$.

Proposition 1 Let s be a real number with $0<s<1$. Then, for any positive even number $n$,

$$
2(n-1)^{s}<A_{n, s}^{-1}<2 n^{s},
$$

and for any positive odd number $n$,

$$
-2 n^{s}<B_{n, s}^{-1}<-2(n-1)^{s}
$$

Proof Let $n$ be a positive even number. For every positive integer $k$, it is easy to see that

$$
\begin{gathered}
\left(\frac{1}{(n+1+2 k)^{s}}-\frac{1}{(n+2+2 k)^{s}}\right) \\
<\left(\frac{1}{(n+2 k)^{s}}-\frac{1}{(n+1+2 k)^{s}}\right) \\
<\left(\frac{1}{(n-1+2 k)^{s}}-\frac{1}{(n+2 k)^{s}}\right) .
\end{gathered}
$$

The summations of each term over $k$ give

$$
A_{n+1, s}<A_{n, s}<A_{n-1, s}
$$

and

$$
\frac{1}{2}\left(A_{n+1, s}+A_{n, s}\right)<A_{n, s}<\frac{1}{2}\left(A_{n-1, s}+A_{n, s}\right) .
$$

Therefore, we have

$$
\frac{1}{2 n^{s}}<A_{n, s}<\frac{1}{2(n-1)^{s}}
$$

which gives the first statement.

The second statement can be shown similarly.

Since every proof of the case when $n$ is an odd number is analogous to that of the case when $n$ is an even number, we omit all the proofs of the odd number cases in this paper.

Now we find tighter bounds for $A_{n, s}^{-1}$ and $B_{n, s}^{-1}$.

Proposition 2 Let $s$ be a real number with $0<s<1$. Then, for any positive even number $n$,

$$
2\left(n-\frac{1}{2}\right)^{s}<A_{n, s}^{-1}
$$


and for any positive odd number n,

$$
B_{n, s}^{-1}<-2\left(n-\frac{1}{2}\right)^{s}
$$

Proof Let $n$ be a positive even number. We will show that

$$
A_{n, s}<\frac{1}{2\left(n-\frac{1}{2}\right)^{s}}
$$

Rewriting each of the both sides as a series

$$
A_{n, s}=\sum_{k=\frac{n}{2}}^{\infty}\left(\frac{1}{(2 k)^{s}}-\frac{1}{(2 k+1)^{s}}\right)
$$

and

$$
\frac{1}{2\left(n-\frac{1}{2}\right)^{s}}=\sum_{k=\frac{n}{2}}^{\infty}\left(\frac{1}{2\left(2 k-\frac{1}{2}\right)^{s}}-\frac{1}{2\left(2 k+\frac{3}{2}\right)^{s}}\right),
$$

we will show that for any positive integer $k$,

$$
\frac{1}{(2 k)^{s}}-\frac{1}{(2 k+1)^{s}}<\frac{1}{2\left(2 k-\frac{1}{2}\right)^{s}}-\frac{1}{2\left(2 k+\frac{3}{2}\right)^{s}} .
$$

For this, we let

$$
f(x)=\left(\frac{1}{2\left(2 x-\frac{1}{2}\right)^{s}}-\frac{1}{2\left(2 x+\frac{3}{2}\right)^{s}}\right)-\left(\frac{1}{(2 x)^{s}}-\frac{1}{(2 x+1)^{s}}\right)
$$

and will show that $f(x)$ is positive for $x \geq 1$ and $0<s<1$. With

$$
g(x)=\frac{1}{2\left(2 x-\frac{1}{2}\right)^{s}}+\frac{1}{2\left(2 x+\frac{1}{2}\right)^{s}}-\frac{1}{(2 x)^{s}}
$$

we have $f(x)=g(x)-g\left(x+\frac{1}{2}\right)$. Consider the derivative of $g(x)$ :

$$
g^{\prime}(x)=-2 s\left(\frac{1}{2\left(2 x-\frac{1}{2}\right)^{s+1}}+\frac{1}{2\left(2 x+\frac{1}{2}\right)^{s+1}}-\frac{1}{(2 x)^{s+1}}\right) .
$$

Since the function $\frac{1}{x^{s+1}}$ is convex, we obtain that

$$
\frac{1}{2\left(2 x-\frac{1}{2}\right)^{s+1}}+\frac{1}{2\left(2 x+\frac{1}{2}\right)^{s+1}}-\frac{1}{(2 x)^{s+1}} \geq 0
$$

and therefore $g^{\prime}(x)$ is negative, that is, $g(x)$ is decreasing. We conclude that $f(x)$ is positive, which gives the statement. 
Proposition 3 Let s be a real number with $0<s<1$. Then, for any positive even number $n$,

$$
A_{n, s}^{-1}<2\left(n-\frac{1}{4}\right)^{s}
$$

and for any positive odd number $n$,

$$
-2\left(n-\frac{1}{4}\right)^{s}<B_{n, s}^{-1}
$$

Proof Let $n$ be a positive even number. We will show that

$$
\frac{1}{2\left(n-\frac{1}{4}\right)^{s}}<A_{n, s}
$$

Rewriting each of the both sides as a series

$$
A_{n, s}=\sum_{k=\frac{n}{2}}^{\infty}\left(\frac{1}{(2 k)^{s}}-\frac{1}{(2 k+1)^{s}}\right)
$$

and

$$
\frac{1}{2\left(n-\frac{1}{4}\right)^{s}}=\sum_{k=\frac{n}{2}}^{\infty}\left(\frac{1}{2\left(2 k-\frac{1}{4}\right)^{s}}-\frac{1}{2\left(2 k+\frac{7}{4}\right)^{s}}\right),
$$

we need to show that for any positive integer $k$,

$$
\frac{1}{2\left(2 k-\frac{1}{4}\right)^{s}}-\frac{1}{2\left(2 k+\frac{7}{4}\right)^{s}}<\frac{1}{(2 k)^{s}}-\frac{1}{(2 k+1)^{s}} .
$$

For this, we let

$$
f(x)=\left(\frac{1}{(2 x)^{s}}-\frac{1}{(2 x+1)^{s}}\right)-\left(\frac{1}{2\left(2 x-\frac{1}{4}\right)^{s}}-\frac{1}{2\left(2 x+\frac{7}{4}\right)^{s}}\right) .
$$

We check that $f(1)>0$ and now we will show that $f(x)$ is positive for $x \geq 2$ and $0<s<1$. With

$$
g(x)=\frac{1}{(2 x)^{s}}-\left(\frac{1}{2\left(2 x-\frac{1}{4}\right)^{s}}+\frac{1}{2\left(2 x+\frac{3}{4}\right)^{s}}\right)
$$

we have $f(x)=g(x)-g\left(x+\frac{1}{2}\right)$, so we only need to show that $g(x)$ is decreasing. Consider the derivative of $g(x)$ :

$$
\begin{aligned}
g^{\prime}(x) & =s\left(-\frac{2}{(2 x)^{s+1}}+\left(\frac{1}{\left(2 x-\frac{1}{4}\right)^{s+1}}+\frac{1}{\left(2 x+\frac{3}{4}\right)^{s+1}}\right)\right) \\
& =s\left(\left(\frac{1}{\left(2 x-\frac{1}{4}\right)^{s+1}}-\frac{1}{(2 x)^{s+1}}\right)-\left(\frac{1}{(2 x)^{s+1}}-\frac{1}{\left(2 x+\frac{3}{4}\right)^{s+1}}\right)\right) .
\end{aligned}
$$


Since the function $\frac{1}{x^{s+1}}$ is decreasing and convex, by comparing slopes at $\left(2 x-\frac{1}{4}\right)$ and $\left(2 x+\frac{3}{4}\right)$, we obtain

$$
\frac{1}{\left(2 x-\frac{1}{4}\right)^{s+1}}-\frac{1}{(2 x)^{s+1}}<\frac{1}{4}(s+1) \frac{1}{\left(2 x-\frac{1}{4}\right)^{s+2}}
$$

and

$$
\frac{1}{(2 x)^{s+1}}-\frac{1}{\left(2 x+\frac{3}{4}\right)^{s+1}}>\frac{1}{4}(s+1) \frac{3}{\left(2 x+\frac{3}{4}\right)^{s+2}} .
$$

Therefore,

$$
g^{\prime}(x)<\frac{1}{4} s(s+1)\left(\frac{1}{\left(2 x-\frac{1}{4}\right)^{s+2}}-\frac{3}{\left(2 x+\frac{3}{4}\right)^{s+2}}\right) .
$$

Consider $h(x, s):=\frac{1}{3}\left(\frac{2 x+3 / 4}{2 x-1 / 4}\right)^{s+2}$, which is the ratio of two terms on the right-hand side of the above expression. We check that $h(x, s)<1$ for $x \geq 2$ and $0<s<1$. Since $h(2,1)=$ $6859 / 10,125$ and $\lim _{x \rightarrow \infty} h(x, s)=\frac{1}{3}$ for $0<s<1$, we obtain that $g^{\prime}(x)$ is negative and, therefore, $g(x)$ is decreasing, which gives the statement.

We combine the results of Proposition 2 and Proposition 3.

Theorem 1 Let s be a real number with $0<s<1$. Then, for any positive even number $n$,

$$
2\left(n-\frac{1}{2}\right)^{s}<A_{n, s}^{-1}<2\left(n-\frac{1}{4}\right)^{s}
$$

and for any positive odd number $n$,

$$
-2\left(n-\frac{1}{4}\right)^{s}<B_{n, s}^{-1}<-2\left(n-\frac{1}{2}\right)^{s} .
$$

We express these bounds in terms of $\zeta_{n}(s)$ using expression (1).

Corollary 1 Let $s$ be a real number with $0<s<1$. Then, for any positive even number $n$,

$$
2\left(1-2^{1-s}\right)\left(n-\frac{1}{4}\right)^{s}<\zeta_{n}(s)^{-1}<2\left(1-2^{1-s}\right)\left(n-\frac{1}{2}\right)^{s},
$$

and for any positive odd number $n$,

$$
-2\left(1-2^{1-s}\right)\left(n-\frac{1}{2}\right)^{s}<\zeta_{n}(s)^{-1}<-2\left(1-2^{1-s}\right)\left(n-\frac{1}{4}\right)^{s} .
$$

Furthermore, we have tighter bounds of $A_{n, s}^{-1}$ and $B_{n, s}^{-1}$ for a sufficiently large number $n$.

Theorem 2 For any positive number $\epsilon$ and any real number $s$ with $0<s<1$,

$$
2\left(n-\frac{1}{2}\right)^{s}<A_{n, s}^{-1}<2\left(n-\frac{1}{2}+\epsilon\right)^{s}
$$


for a sufficiently large even number $n$ and

$$
-2\left(n-\frac{1}{2}+\epsilon\right)^{s}<B_{n, s}^{-1}<-2\left(n-\frac{1}{2}\right)^{s}
$$

for a sufficiently large odd number $n$.

Proof From Theorem 1, it suffices to show that for a sufficiently large even number $n$,

$$
\frac{1}{2\left(n-\frac{1}{2}+\epsilon\right)^{s}}<A_{n, s}
$$

Rewriting each of the both sides as a series

$$
A_{n, s}=\sum_{k=\frac{n}{2}}^{\infty}\left(\frac{1}{(2 k)^{s}}-\frac{1}{(2 k+1)^{s}}\right)
$$

and

$$
\frac{1}{2\left(n-\frac{1}{2}+\epsilon\right)^{s}}=\sum_{k=\frac{n}{2}}^{\infty}\left(\frac{1}{2\left(2 k-\frac{1}{2}+\epsilon\right)^{s}}-\frac{1}{2\left(2 k+\frac{3}{2}+\epsilon\right)^{s}}\right),
$$

we need to show that for a sufficiently large even number $n$ and every integer $k \geq \frac{n}{2}$,

$$
\frac{1}{2\left(2 k-\frac{1}{2}+\epsilon\right)^{s}}-\frac{1}{2\left(2 k+\frac{3}{2}+\epsilon\right)^{s}}<\frac{1}{(2 k)^{s}}-\frac{1}{(2 k+1)^{s}} .
$$

For this, let

$$
f(x)=\left(\frac{1}{(2 x)^{s}}-\frac{1}{(2 x+1)^{s}}\right)-\left(\frac{1}{2\left(2 x-\frac{1}{2}+\epsilon\right)^{s}}-\frac{1}{2\left(2 x+\frac{3}{2}+\epsilon\right)^{s}}\right),
$$

and we will show that $f(x)$ is positive for $x \geq x_{0}$, where $x_{0}$ is a sufficiently large number. With

$$
g(x)=\frac{1}{(2 x)^{s}}-\left(\frac{1}{2\left(2 x-\frac{1}{2}+\epsilon\right)^{s}}+\frac{1}{2\left(2 x+\frac{1}{2}+\epsilon\right)^{s}}\right),
$$

we have that $f(x)=g(x)-g\left(x+\frac{1}{2}\right)$, so we only need to show that $g(x)$ is decreasing. Consider the derivative of $g(x)$ :

$$
\begin{aligned}
g^{\prime}(x) & =s\left(-\frac{2}{(2 x)^{s+1}}+\frac{1}{\left(2 x-\frac{1}{2}+\epsilon\right)^{s+1}}+\frac{1}{\left(2 x+\frac{1}{2}+\epsilon\right)^{s+1}}\right) \\
& =s\left(\left(\frac{1}{\left(2 x-\frac{1}{2}+\epsilon\right)^{s+1}}-\frac{1}{(2 x)^{s+1}}\right)-\left(\frac{1}{(2 x)^{s+1}}-\frac{1}{\left(2 x+\frac{1}{2}+\epsilon\right)^{s+1}}\right)\right) .
\end{aligned}
$$

Since $\frac{1}{x^{s+1}}$ is decreasing and convex, by comparing slopes at $\left(2 x-\frac{1}{2}+\epsilon\right)$ and $\left(2 x+\frac{1}{2}+\epsilon\right)$, we obtain

$$
\frac{1}{\left(2 x-\frac{1}{2}+\epsilon\right)^{s+1}}-\frac{1}{(2 x)^{s+1}}<(s+1) \frac{\frac{1}{2}-\epsilon}{\left(2 x-\frac{1}{2}+\epsilon\right)^{s+2}}
$$


and

$$
\frac{1}{(2 x)^{s+1}}-\frac{1}{\left(2 x+\frac{1}{2}+\epsilon\right)^{s+1}}>(s+1) \frac{\frac{1}{2}+\epsilon}{\left(2 x+\frac{1}{2}+\epsilon\right)^{s+2}} .
$$

Therefore

$$
g^{\prime}(x)<s(s+1)\left(\frac{\frac{1}{2}-\epsilon}{\left(2 x-\frac{1}{2}+\epsilon\right)^{s+2}}-\frac{\frac{1}{2}+\epsilon}{\left(2 x+\frac{1}{2}+\epsilon\right)^{s+2}}\right) .
$$

Consider $h(x):=\frac{\frac{1}{2}-\epsilon}{\frac{1}{2}+\epsilon}\left(\frac{2 x+\frac{1}{2}+\epsilon}{2 x-\frac{1}{2}+\epsilon}\right)^{s+2}$, which is the ratio of two terms on the right-hand side of the above expression. We need to show that $h(x)<1$ for every $x>x_{0}$, where $x_{0}$ is a sufficiently large number. We check that

$$
h(x)<1 \Longleftrightarrow \frac{2 x+\frac{1}{2}+\epsilon}{2 x-\frac{1}{2}+\epsilon}<\left(\frac{\frac{1}{2}+\epsilon}{\frac{1}{2}-\epsilon}\right)^{\frac{1}{s+2}} .
$$

For any $\epsilon>0$ and $0<s<1$, we have that $1<\left(\frac{\frac{1}{2}+\epsilon}{\frac{1}{2}-\epsilon}\right)^{1 /(s+2)}$ and $\frac{2 x+\frac{1}{2}+\epsilon}{2 x-\frac{1}{2}+\epsilon}$ is larger than 1 , decreasing and converges to 1 as $x$ goes to infinity, so there is $x_{0}$ such that, for every $x>x_{0}$, $h(x)<1$. Therefore the proof is complete.

We express these bounds in terms of $\zeta_{n}(s)$ using expression (1).

Corollary 2 For any positive number $\epsilon$ and any real number $s$ with $0<s<1$, we have

$$
2\left(1-2^{1-s}\right)\left(n-\frac{1}{2}+\epsilon\right)^{s}<\zeta_{n}(s)^{-1}<2\left(1-2^{1-s}\right)\left(n-\frac{1}{2}\right)^{s}
$$

for a sufficiently large even number $n$ and

$$
-2\left(1-2^{1-s}\right)\left(n-\frac{1}{2}\right)^{s}<\zeta_{n}(s)^{-1}<-2\left(1-2^{1-s}\right)\left(n-\frac{1}{2}+\epsilon\right)^{s}
$$

for a sufficiently large odd number $n$.

\subsection{The value of the inverse of $\zeta_{n}(s)$ for $s=\frac{1}{2}, \frac{1}{3}$, and $\frac{1}{4}$}

We study firstly the value of the inverse of $\zeta_{n}\left(\frac{1}{2}\right)$, where $\zeta_{n}\left(\frac{1}{2}\right)$ is the tail of the Riemann zeta function from $n$ at $s=\frac{1}{2}$.

Theorem 3 For any positive even number $n$,

$$
\left[A_{n, 1 / 2}^{-1}\right]=\left[2\left(n-\frac{1}{2}\right)^{1 / 2}\right]
$$

and for any positive odd number $n$,

$$
\left[B_{n, 1 / 2}^{-1}\right]=\left[-2\left(n-\frac{1}{2}\right)^{1 / 2}\right]
$$


Proof Let $n$ be a positive even number. By Theorem 1, we have that

$$
2\left(n-\frac{1}{2}\right)^{1 / 2}<A_{n, 1 / 2}^{-1}<2\left(n-\frac{1}{4}\right)^{1 / 2}
$$

Note that $2\left(n-\frac{1}{4}\right)^{1 / 2}-2\left(n-\frac{1}{2}\right)^{1 / 2}<1$ for $n \geq 2$, and it implies that there is at most one integer in the open interval from $2\left(n-\frac{1}{2}\right)^{1 / 2}$ to $2\left(n-\frac{1}{4}\right)^{1 / 2}$. Suppose that there is an integer $h$ in the open interval, i.e.,

$$
2\left(n-\frac{1}{2}\right)^{1 / 2}<h<2\left(n-\frac{1}{4}\right)^{1 / 2} \text { or } \quad 4 n-2<h^{2}<4 n-1 .
$$

There is, however, no integer in the open interval from $4 n-2$ to $4 n-1$, therefore such an integer $h$ does not exist. This gives the statement.

We express this result in terms of $\zeta_{n}(s)$ using expression (1).

Corollary 3 For any positive integer $n$,

$$
\left[\frac{1}{1-2^{1 / 2}} \zeta_{n}\left(\frac{1}{2}\right)^{-1}\right]=\left[(-1)^{n+1} 2\left(n-\frac{1}{2}\right)^{1 / 2}\right]
$$

We study secondly the value of the inverse of $\zeta_{n}\left(\frac{1}{3}\right)$, where $\zeta_{n}\left(\frac{1}{3}\right)$ is the tail of the Riemann zeta function from $n$ at $s=\frac{1}{3}$.

Theorem 4 For any positive even number $n$,

$$
\left[A_{n, 1 / 3}^{-1}\right]=\left[2\left(n-\frac{1}{2}\right)^{1 / 3}\right]
$$

and for any positive odd number $n$,

$$
\left[B_{n, 1 / 3}^{-1}\right]=\left[-2\left(n-\frac{1}{2}\right)^{1 / 3}\right]
$$

Proof Let $n$ be a positive even number. By Theorem 1, we have that

$$
2\left(n-\frac{1}{2}\right)^{1 / 3}<A_{n, 1 / 3}^{-1}<2\left(n-\frac{1}{4}\right)^{1 / 3}
$$

Note that $2\left(n-\frac{1}{4}\right)^{1 / 3}-2\left(n-\frac{1}{2}\right)^{1 / 3}<1$ for $n \geq 2$, and it implies that there is at most one integer in the open interval from $2\left(n-\frac{1}{2}\right)^{1 / 3}$ to $2\left(n-\frac{1}{4}\right)^{1 / 3}$. Suppose that there is an integer $h$ in the open interval, i.e.,

$$
2\left(n-\frac{1}{2}\right)^{1 / 3}<h<2\left(n-\frac{1}{4}\right)^{1 / 3} \quad \text { or } \quad 8 n-4<h^{3}<8 n-2 .
$$


This shows that the integer $h$ is of the form $h=2\left(n-\frac{3}{8}\right)^{1 / 3}$. If we show $A_{n, 1 / 3}^{-1}<2\left(n-\frac{3}{8}\right)^{1 / 3}$ or, equivalently, $\frac{1}{2\left(n-\frac{3}{8}\right)^{1 / 3}}<A_{n, 1 / 3}$, then our proof will be done. Let us rewrite

$$
A_{n, 1 / 3}=\sum_{k=\frac{n}{2}}^{\infty}\left(\frac{1}{(2 k)^{1 / 3}}-\frac{1}{(2 k+1)^{1 / 3}}\right)
$$

and

$$
\frac{1}{2\left(n-\frac{3}{8}\right)^{1 / 3}}=\sum_{k=\frac{n}{2}}^{\infty}\left(\frac{1}{2\left(2 k-\frac{3}{8}\right)^{1 / 3}}-\frac{1}{2\left(2 k+\frac{13}{8}\right)^{1 / 3}}\right) .
$$

Now it suffices to show that for any positive integer $k$,

$$
\frac{1}{2\left(2 k-\frac{3}{8}\right)^{1 / 3}}-\frac{1}{2\left(2 k+\frac{13}{8}\right)^{1 / 3}}<\frac{1}{(2 k)^{1 / 3}}-\frac{1}{2(2 k+1)^{1 / 3}} .
$$

For this, we let

$$
f(x)=\left(\frac{1}{(2 x)^{1 / 3}}-\frac{1}{(2 x+1)^{1 / 3}}\right)-\left(\frac{1}{2\left(2 x-\frac{3}{8}\right)^{1 / 3}}-\frac{1}{2\left(2 x+\frac{13}{8}\right)^{1 / 3}}\right),
$$

and we will show that $f(x)$ is positive for any positive integer $x$.

We check that $f(1)=0.00053 \cdots$ and $f(2)=0.00081 \cdots$, so it suffices to show $f(x)>0$ for $x \geq 3$. With

$$
g(x)=\frac{1}{(2 x)^{1 / 3}}-\left(\frac{1}{2\left(2 x-\frac{3}{8}\right)^{1 / 3}}+\frac{1}{2\left(2 x+\frac{5}{8}\right)^{1 / 3}}\right),
$$

we have that $f(x)=g(x)-g\left(x+\frac{1}{2}\right)$, so we only need to show that $g(x)$ is decreasing for $x \geq 3$. Consider the derivative of $g(x)$ :

$$
\begin{aligned}
g^{\prime}(x) & =\frac{1}{3}\left(-\frac{2}{(2 x)^{4 / 3}}+\frac{1}{\left(2 x-\frac{3}{8}\right)^{4 / 3}}+\frac{1}{\left(2 x+\frac{5}{8}\right)^{4 / 3}}\right) \\
& =\frac{1}{3}\left(\left(\frac{1}{\left(2 x-\frac{3}{8}\right)^{4 / 3}}-\frac{1}{(2 x)^{4 / 3}}\right)-\left(\frac{1}{(2 x)^{4 / 3}}-\frac{1}{\left(2 x+\frac{5}{8}\right)^{4 / 3}}\right)\right) .
\end{aligned}
$$

Since $\frac{1}{x^{4 / 3}}$ is decreasing and convex, by comparing slopes at $\left(2 x-\frac{3}{8}\right)$ and $\left(2 x+\frac{5}{8}\right)$, we obtain

$$
\frac{1}{\left(2 x-\frac{3}{8}\right)^{4 / 3}}-\frac{1}{(2 x)^{4 / 3}}<2 \cdot \frac{3}{16} \cdot \frac{4}{3} \cdot \frac{1}{\left(2 x-\frac{3}{8}\right)^{7 / 3}}
$$

and

$$
\frac{1}{(2 x)^{4 / 3}}-\frac{1}{\left(2 x+\frac{5}{8}\right)^{4 / 3}}>2 \cdot \frac{5}{16} \cdot \frac{4}{3} \cdot \frac{1}{\left(2 x+\frac{5}{8}\right)^{7 / 3}} .
$$

Therefore

$$
g^{\prime}(x)<\frac{1}{18}\left(\frac{3}{\left(2 x-\frac{3}{8}\right)^{7 / 3}}-\frac{5}{\left(2 x+\frac{5}{8}\right)^{7 / 3}}\right) .
$$


Consider $h(x):=\frac{3}{5}\left(\frac{2 x+5 / 8}{2 x-3 / 8}\right)^{7 / 3}$, which is the ratio of two terms of the right-hand side of the above expression. We check that $h(x)<1$ for $x \geq 3$ because $h(3)=0.87 \cdots$ and $\lim _{x \rightarrow \infty} h(x)=\frac{3}{5}$ and $h^{\prime}(x)<0$ for $x \geq 3$. Hence we obtain that $g^{\prime}(x)$ is negative and so $g(x)$ is decreasing for $x \geq 3$, which proves the statement.

We express this result in terms of $\zeta_{n}(s)$ using expression (1).

Corollary 4 For any positive integer $n$,

$$
\left[\frac{1}{1-2^{2 / 3}} \zeta_{n}\left(\frac{1}{3}\right)^{-1}\right]=\left[(-1)^{n+1} 2\left(n-\frac{1}{2}\right)^{1 / 3}\right] \text {. }
$$

We study lastly the value of the inverse of $\zeta_{n}\left(\frac{1}{4}\right)$, which is the tail of the Riemann zeta function from $n$ at $s=\frac{1}{4}$.

Theorem 5 For any positive even number $n$,

$$
\left[A_{n, 1 / 4}^{-1}\right]=\left[2\left(n-\frac{1}{2}\right)^{1 / 4}\right]
$$

and for any positive odd number $n$,

$$
\left[B_{n, 1 / 4}^{-1}\right]=\left[-2\left(n-\frac{1}{2}\right)^{1 / 4}\right]
$$

Proof Let $n$ be a positive even number. By Theorem 1, we have that

$$
2\left(n-\frac{1}{2}\right)^{1 / 4}<A_{n, 1 / 4}^{-1}<2\left(n-\frac{1}{4}\right)^{1 / 4} .
$$

Note that $2\left(n-\frac{1}{4}\right)^{1 / 4}-2\left(n-\frac{1}{2}\right)^{1 / 4}<1$ for $n \geq 2$, and it implies that there is at most one integer in the open interval from $2\left(n-\frac{1}{2}\right)^{1 / 4}$ to $2\left(n-\frac{1}{4}\right)^{1 / 4}$. Suppose that there is an integer $h$ in the open interval, i.e.,

$$
2\left(n-\frac{1}{2}\right)^{1 / 4}<h<2\left(n-\frac{1}{4}\right)^{1 / 4} \text { or } 16 n-8<h^{4}<16 n-4 .
$$

This shows that the integer $h^{4}$ is one of the form $16 n-7,16 n-6$, or $16 n-5$. For any integer $h$, however, $h^{4} \equiv 0$ or 1 ( $\left.\bmod 16\right)$, hence such an integer $h$ does not exist. Therefore this gives the statement.

We express this result in terms of $\zeta_{n}(s)$ using expression (1).

Corollary 5 For any positive integer $n$,

$$
\left[\frac{1}{1-2^{3 / 4}} \zeta_{n}\left(\frac{1}{4}\right)^{-1}\right]=\left[(-1)^{n+1} 2\left(n-\frac{1}{2}\right)^{1 / 4}\right] \text {. }
$$

We express the results of Theorems 3,4 , and 5 in a single statement. 
Theorem 6 For $s=\frac{1}{2}, \frac{1}{3}$, or $\frac{1}{4}$, and for any positive even number $n$,

$$
\left[A_{n, s}^{-1}\right]=\left[2\left(n-\frac{1}{2}\right)^{s}\right]
$$

and for any positive odd number $n$,

$$
\left[B_{n, s}^{-1}\right]=\left[-2\left(n-\frac{1}{2}\right)^{s}\right]
$$

We express the results of Corollaries 3, 4, and 5 in a single statement.

Corollary 6 For any positive integer $n$ and $s=\frac{1}{2}, \frac{1}{3}$, or $\frac{1}{4}$,

$$
\left[\frac{1}{1-2^{1-s}} \zeta_{n}(s)^{-1}\right]=\left[(-1)^{n+1} 2\left(n-\frac{1}{2}\right)^{s}\right] .
$$

\section{Conclusion}

In this paper, we have presented the bounds of $A_{n, s}^{-1}$ and $B_{n, s}^{-1}$, hence the bounds of the inverses of tails of the Riemann zeta function $\zeta_{n}(s)^{-1}$ for $0<s<1$, and computed the values $\left[A_{n, s}^{-1}\right]$ and $\left[B_{n, s}^{-1}\right]$, hence the values of the inverses of tails of the Riemann zeta function $\left[\frac{1}{1-2^{1-s}} \zeta_{n}(s)^{-1}\right]$ for $s=\frac{1}{2}, \frac{1}{3}$, and $\frac{1}{4}$. For other values of $s$, for example $s=\frac{1}{5}$ or $\frac{2}{3}$, the values of $A_{n, s}$ and $B_{n, s}$ do not seem to have simple expressions.

\section{Acknowledgements}

The authors would like to express their thanks to the referees and the editors for their helpful comments and advice.

\section{Funding}

We have not received any funding.

\section{Abbreviations}

Not applicable.

Availability of data and materials

Not applicable.

\section{Competing interests}

We declare that none of the authors has any competing interests in the manuscript.

\section{Authors' contributions}

All authors equally contributed to the manuscript. All authors read and approved the final manuscript.

\section{Publisher's Note}

Springer Nature remains neutral with regard to jurisdictional claims in published maps and institutional affiliations.

Received: 27 March 2018 Accepted: 20 June 2018 Published online: 03 July 2018

\section{References}

1. Borwein, P., Choi, S., Rooney, B., Weirathmuellerer, A.: The Riemann Hypothesis. CMS Books in Mathematics/Ouvrages de Mathématiques de la SMC. Springer, New York (2008)

2. Euler, L.: Variae observationes circa series infinitas. Commentarii academiae scientiarum Petropolitanae 9, 160-188 (1744). (Presented to the St. Petersburg Academy in 1737) = Opera Omnia Ser. 114, 217-244. https://www.biodiversitylibrary.org

3. Hwang, W., Song, K.: A reciprocal sum related to the Riemann zeta function at $s=6$. Preprint. https://arxiv.org/abs/1709.07994

4. Luo, Q.-M., Guo, B.-N., Qi, F.: On evaluation of Riemann zeta function $\zeta$ (s). Adv. Stud. Contemp. Math. (Kyungshang) 7(2), 135-144 (2003)

5. Luo, Q.-M., Wei, Z.-L., Qi, F.: Lower and upper bounds of $\zeta$ (3). Adv. Stud. Contemp. Math. (Kyungshang) 6(1), 47-51 (2003) 
6. Riemann, B.: Über die Anzahl der Primzahlen unter einer gegebenen Größe. Monatsberichte der Berliner Akademie, 671-680 (1859)

7. Xin, L.: Some identities related to Riemann zeta-function. J. Inequal. Appl. 2016(1), 32 (2016). https://doi.org/10.1186/s13660-016-0980-9

8. Xin, L., Xiaoxue, L.: A reciprocal sum related to the Riemann $\zeta$ - function. J. Math. Inequal. 11(1), $209-215$ (2017). https://doi.org/10.7153/jmi-11-20

9. Xu, H.: Some computational formulas related to the Riemann zeta-function tails. J. Inequal. Appl. 2016(1), 132 (2016), https://doi.org/10.1186/s13660-016-1068-2

Submit your manuscript to a SpringerOpen ${ }^{\circ}$ journal and benefit from:

- Convenient online submission

$\checkmark$ Rigorous peer review

Open access: articles freely available online

- High visibility within the field

- Retaining the copyright to your article

Submit your next manuscript at $\gg$ springeropen.com 